\title{
A novel hybrid approach for level set characterization and tracking of non-planar 3D cracks in the extended finite element method
}

\author{
Alireza Sadeghirad ${ }^{1}$, David L Chopp ${ }^{2}$, Xiang Ren ${ }^{1}$, Eugene Fang ${ }^{1}$, and Jim Lua ${ }^{1 *}$ \\ ${ }^{1}$ Global Engineering and Materials, Inc., Princeton, NJ 08540, USA \\ ${ }^{2}$ Department of Engineering Sciences and Applied Mathematics, Northwestern University, Evanston, IL 60208, USA
}

Elsevier use only: Received date here; revised date here; accepted date here

\begin{abstract}
In this paper, the limitations associated with implicit and explicit representations of cracks in the extended finite element method (XFEM) is recapitulated via numerical explorations followed by the development of a novel hybrid approach for the characterization of non-planar 3D cracks along with its capability demonstrations. In the XFEM, the crack geometry is independent of the structural mesh, and is often described implicitly by means of two level set functions. The implicit representation is very convenient for purposes of computing the crack front velocity and for handling situations where crack front is concave and the velocity vectors may cross. The main difficulty of this implicit description is the formulation of an efficient and robust update scheme for the level set values after a propagation step. On the other hand, the crack geometry can be described by an explicit triangulated mesh which can be easily updated after a propagation step. The explicit representation has its own shortcomings, e.g., difficulties in handling crack overlaps and extraction of crack local coordinates. Given the difficulties associated with the use of either implicit or explicit method for the geometric description of complex crack geometry, a novel hybrid method is developed by a combination of an implicit level set representation of the crack and an explicit triangulated mesh representation. In the hybrid approach, the implicit representation is updated after each propagation step and disconnected crack surfaces are removed using a paint-fill algorithm based on the current explicit representation of the crack. Then, an updated explicit representation is constructed based on the updated implicit representation using the marching cubes algorithm. Finally, the implicit representation is rebuilt from the explicit representation. The use of the explicit representation ensures that the data in the level set representation is generated from a consistent crack description. The effectiveness of the developed hybrid approach is demonstrated by analyzing several 3D crack propagation problems including a quarter-circular crack in a complex helicopter component, a u-shaped crack and an inclined elliptical crack in cuboids, and an inclined edge crack in a three-point bending beam. () 2001 Elsevier Science. All rights reserved
\end{abstract}

Keywords: extended finite element method (XFEM); non-planar 3D cracks; crack propagation; level set characterization; Abaqus

\footnotetext{
${ }^{*}$ Corresponding author. Tel.: +1-609-356-5115; fax: +1-609-924-3999; e-mail: jlua@gem-innovation.com.
} 


\section{Introduction}

The extended finite element method (XFEM) [1,2] has been extensively used to introduce jumps, kinks, singularities, and other nonsmooth features within finite element models independently of the mesh [3]. It is achieved by means of special functions enriching the standard finite element (FE) shape functions. The XFEM has been more and more commonly used for applications in fracture mechanics [4-11]. The XFEM belongs to the category of the continuum-based approach for analysis of solid mechanics problems while the other category, the discontinuous approach, also includes very powerful methods for fracture mechanics simulations, e.g., the peridynamics method [12, 13], and the volume-compensated particle method [14, 15]. Using the discontinuous approach, crack initiation and propagation processes can be modeled in a unified way based on bond breaking and removal rules and they avoid singularity-related issues. On the other hand, the main difficulties of the continuum-based fracture mechanics approach are the stress singularity at the crack front and modeling discontinuities in the displacement field, which requires expensive remeshing in the conventional finite element methods.

Presence of a crack means the discontinuity in the displacement field across the crack surface. Through a local enrichment of the FE shape functions of the elements surrounding the crack, the XFEM introduces this displacement discontinuity and also the singularity of the stress field at the crack front. The locations of the crack surface and front are often defined implicitly in the XFEM by means of the level set method [16-18]. Using the level set method an interface is represented as the zero level set of some higher dimensional function. In the XFEM, two level set functions are used to describe the crack (Fig. 1): 1) the normal level set function, $\varphi(\mathbf{x}, t)$, which gives the signed normal distance between point $\mathbf{x}$ and the crack surface, and 2) the tangential level set function, $\psi(\mathbf{x}, t)$, which gives the signed normal distance between point $\mathbf{x}$ and the surface obtained extruding the crack front normally to the crack surface. The level set values are updated as the crack propagates. Using these two level set functions, the crack front is represented as

$$
\begin{aligned}
& \varphi(\mathbf{x}, t)=0 \\
& \psi(\mathbf{x}, t)=0
\end{aligned}
$$

and the crack surface is given by all the points satisfying the following conditions

$$
\begin{aligned}
& \varphi(\mathbf{x}, t)=0 \\
& \psi(\mathbf{x}, t)<0
\end{aligned}
$$




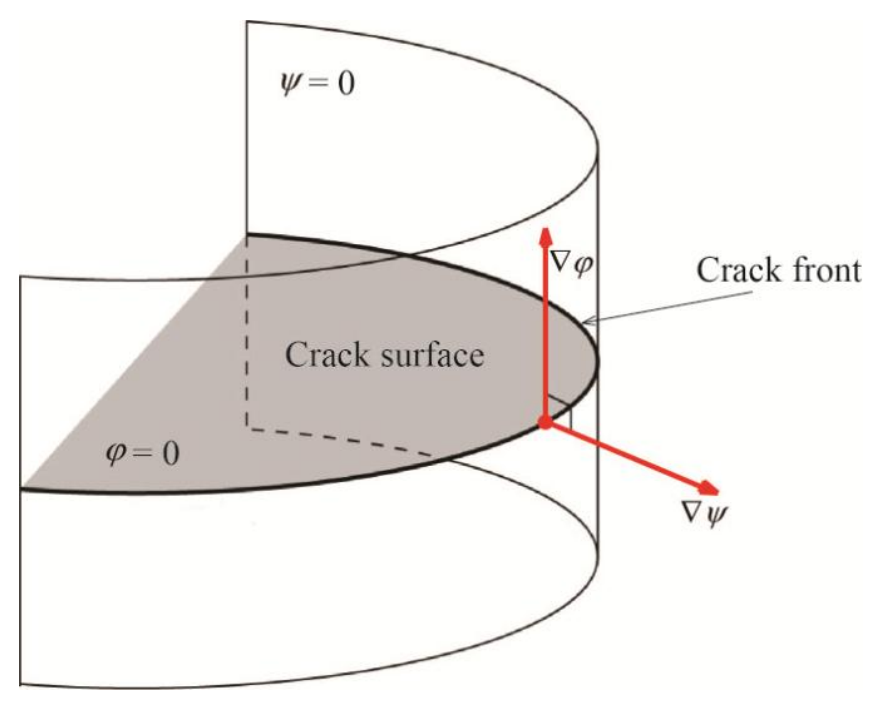

Fig. 1. The implicit representation of a crack in the XFEM using two level set functions.

The level set method and the XFEM is a natural combination of methods where the level set functions maintain the local coordinate system required for the evaluation of the enrichment functions in XFEM. This local coordinate system is used to easily determine which elements are fully/partially cut by the crack, which nodes require enrichment, and in the computation of the stress intensity factors. The level set method is convenient for handling some difficult crack front evolution conditions, for example when the crack front is concave and the velocity vectors may cross as shown in Fig. 2. However, the implicit level set representation also has some drawbacks. The most challenging difficulty is controlling the values of $\varphi$ and $\psi$ so that cracks are not erroneously created in regions disconnected and often far away from the true crack surface. This can happen, for example, when a crack front makes a large change in direction. The values of $\varphi$ and $\psi$ away from the front are determined by extrapolation, and this can lead to unexpected sign changes, which appear in the algorithm as spontaneously created crack surfaces. Use of narrow band techniques can reduce but not entirely eliminate the incidence of these events.

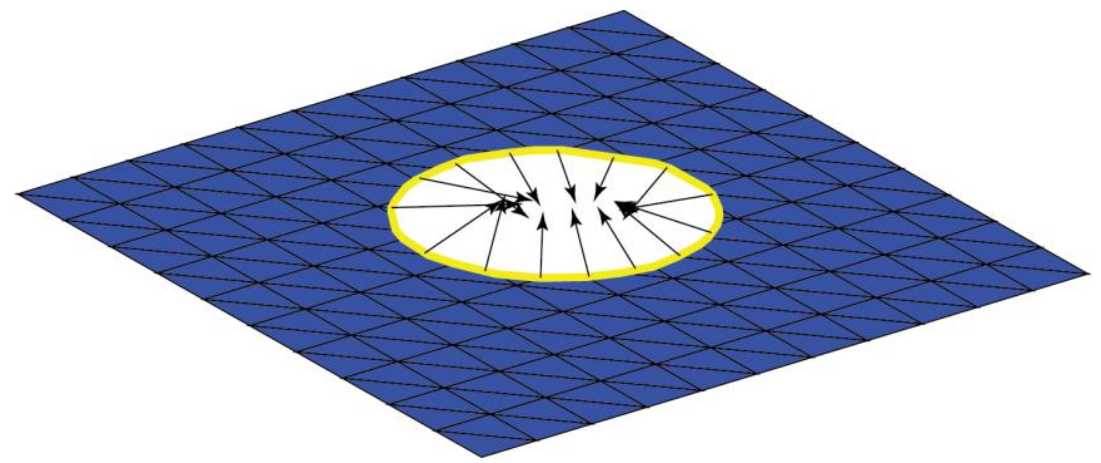

Fig. 2. Example of crossing velocity vectors for a crack that forms a closing circle.

Fig. 3 shows two snapshots of crack propagation in a complex helicopter component using the implicit representation of the crack surface. This example shows when the problem geometry is complex, the implicit approach may lead to creation of spurious crack surfaces in the regions disconnected from the true crack surface. This is mainly due to the erroneous detection of intersections of an extended crack front with multiple boundaries of a solid component. Details about this example are presented in Section 4.1. 


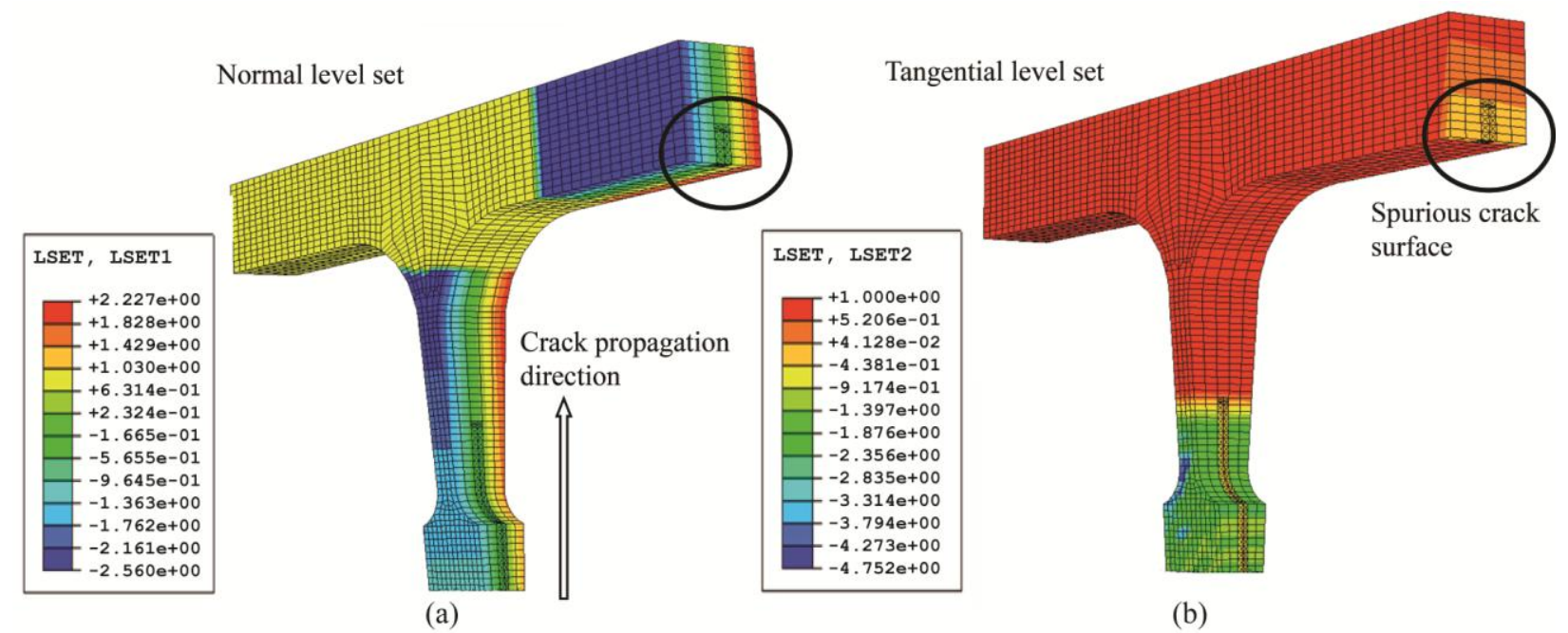

Fig. 3. Two snapshots of the crack propagation from a quarter-circular crack in the helicopter component using the implicit approach, colored based on: (a) the normal level set values and (b) the tangential level set values.

Alternatively, the crack geometry can be described by an explicit triangulated mesh that can be easily updated after a propagation step, and it can clearly demarcate the location of the crack surface and front. But the explicit representation has its own shortcomings, e.g., difficulties in handling crack overlaps and extraction of the local crack coordinates. The explicit representation also presents challenges in the context of treating cracks with concave fronts such as a U-shaped crack shown in Fig. 4. This is mainly due to its inability in evolving a crack front at which its velocity vectors are severely crossed over. Fig. 4 shows three snapshots of crack propagation from a U-shape crack in a cuboid using the explicit crack representation. The simulation failed after seven increments because the new triangles, updated from cross-over propagation vectors, form a non-smooth crack front which prevents continuing smooth crack propagation.

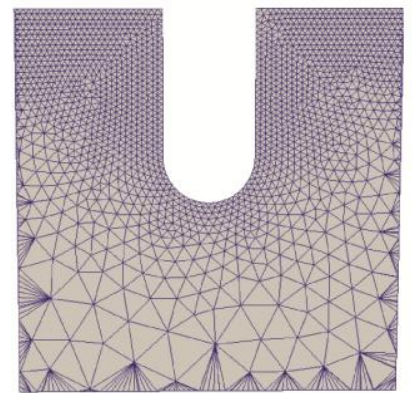

(a)

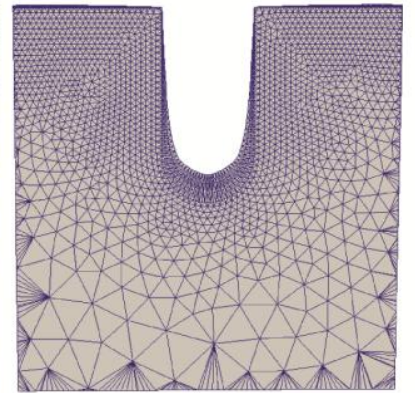

(b)

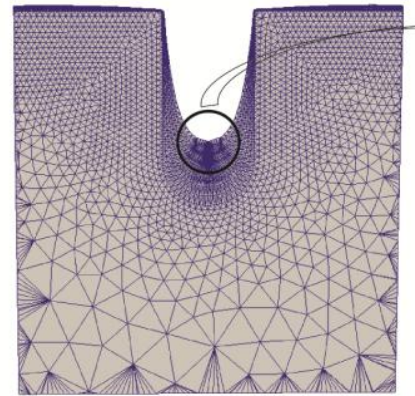

(c)

)

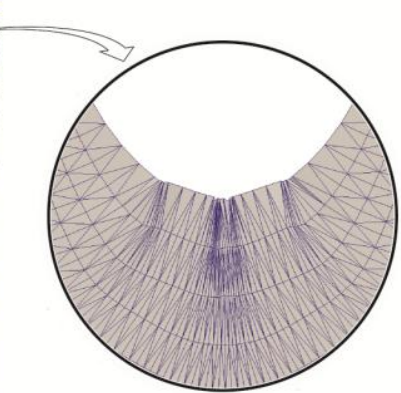

Fig. 4. Three snapshots of the crack propagation from a U-shape crack in a cuboid using the explicit approach: (a) initial configuration, (b) after three crack propagation increments, and (c) after seven crack propagation increments.

The goal of the proposed hybrid explicit/implicit algorithm is to combine the two representations to take advantage of the strengths of each, while simultaneously covering their weaknesses. The explicit representation and its very local description of the crack surface and front can help keep the crack front description localized and not allow the implicit representation to create spurious crack surfaces. On the other hand, the implicit representation can provide the local coordinate 
information and handle merging crack velocity vectors and crack/solid boundary interactions effectively. The key is to merge them in a rational way.

In the proposed approach, the implicit representation is updated directly from the crack front velocity data after each propagation step. The normal level set values are updated only ahead of the crack front. For the updated implicit representation, there is a possibility that new crack surfaces disconnected to the current surface can appear due to extrapolation away from the crack front. We developed some algorithms, discussed in detail in Section 3, to identify if a crack surface is disconnected from the previous crack location and remove it. An updated explicit representation is then constructed based on the updated implicit representation using an adaptation of the marching cubes algorithm [19]. Finally, the implicit representation is rebuilt from the explicit representation. The explicit representation ensures that the data in the level set representation is generated from a consistent crack description. The proposed hybrid approach has been implemented in a 3D extended finite element toolkit for Abaqus (XFA3D) [11].

We note that Fries and Baydoun [20] already developed a hybrid explicit/implicit crack description for the XFEM simulation of crack propagation. In their method, the explicit description of the crack is updated directly based on the crack velocity, and the level set values are updated using the new explicit description. Their method differs from ours in that we update the implicit description based on the crack velocity and use the explicit description to ensure that the data in the level set representation is generated from a consistent crack description.

The outline of this paper is as follows: in Section (2), we briefly review the XFEM formulation. The proposed hybrid approach for tracking of non-planar 3D cracks in the XFEM framework is discussed in Section (3). The effectiveness of the proposed approach in non-planar crack propagation is demonstrated through some numerical examples in Section (4), followed by some conclusions made in Section (5).

\section{XFEM formulation}

The standard version of the XFEM approximation is given by

$$
u^{h}(\xi, \eta, \varsigma)=\sum_{i} N_{i}(\xi, \eta, \varsigma) U_{i}+\sum_{i} N_{i}(\xi, \eta, \varsigma) H(\xi, \eta, \varsigma) b_{i}+\sum_{i} N_{i}(\xi, \eta, \varsigma)\left(\sum_{j=1}^{4} \Psi_{j}(r, \theta) c_{j i}\right)
$$

where $N_{i}(\xi, \eta, \varsigma)$ are the shape functions, $U_{i}$ are the nodal displacement parameters for all the nodes, $b_{i}$ are the jump function parameters at the jump-enriched nodes, $c_{j i}$ are the branch function parameters at the front-enriched nodes, $H(\xi, \eta, \varsigma)$ is the jump function, and $\Psi_{j}(r, \theta)$ are the branch functions.

The jump function is defined as the sign of the normal level set value

$$
H(\xi, \eta, \varsigma)= \begin{cases}+1, & \varphi(\xi, \eta, \varsigma)>0 \\ -1, & \varphi(\xi, \eta, \varsigma)<0\end{cases}
$$


The branch functions, which represent the singular stresses and strains at the crack front, are defined on a polar coordinate system $(r, \theta)$ where $\theta$ is the angle to the tangent plane at the front and $r$ is the distance from the front:

$$
\begin{array}{ll}
\Psi_{1}(r, \theta)=\sqrt{r} \sin (\theta / 2), & \Psi_{2}(r, \theta)=\sqrt{r} \cos (\theta / 2) \\
\Psi_{3}(r, \theta)=\sqrt{r} \sin (\theta / 2) \sin (\theta), & \Psi_{4}(r, \theta)=\sqrt{r} \cos (\theta / 2) \sin (\theta)
\end{array}
$$

where

$$
r=\sqrt{\varphi^{2}(\xi, \eta, \varsigma)+\psi^{2}(\xi, \eta, \varsigma)}, \quad \theta=\operatorname{atan}\left(\frac{\varphi(\xi, \eta, \varsigma)}{\psi(\xi, \eta, \varsigma)}\right)
$$

The level set fields $\varphi(\xi, \eta, \varsigma)$ and $\psi(\xi, \eta, \varsigma)$ are interpolated from their values using the FE shape functions

$$
\begin{aligned}
& \varphi(\xi, \eta, \varsigma)=\sum_{i} N_{i}(\xi, \eta, \varsigma) \varphi_{i} \\
& \psi(\xi, \eta, \varsigma)=\sum_{i} N_{i}(\xi, \eta, \varsigma) \psi_{i}
\end{aligned}
$$

In the XFEM, the approximated displacement field (Eq. (3)) is composed of three terms: the first is a classical FEM approximation composed of the FE shape functions and nodal displacement parameters. Two additional enrichments terms are also present. The second term on the righthand side of Eq. (3) is based on the jump enrichment function and takes the discontinuity in the displacement fields across the crack path/surface into account. Finally, the last term is based on the four branch functions and takes into account the singularity enrichment for the singular stresses and strains at the crack front.

\section{Hybrid approach for level set characterization and tracking of non-planar 3D cracks}

In this section, at first we discuss the implicit and explicit representations of crack surface used in this paper and then, the proposed hybrid implicit/explicit representation is discussed in details.

\subsection{The implicit approach for tracking cracks}

In the implicit approach, the nodal level set values are defined on a regular rectilinear grid, which does not have to conform to the crack or solid domain shape. The level set values in the implicit representation can be directly updated from the crack front velocity data which are obtained based on the stress intensity factors at the sampling points along the crack front. The optimum grid spacing, based on the numerical simulations, is about half the FE mesh size. Using coarse grids may lead to inaccurate results and very refined grids require high computational costs without notably improving the results. In the proposed hybrid implicit/explicit approach, we do not directly update the explicit representation to avoid the issue of crossing velocity vectors. Direct update of the implicit representation advances the crack without this trap. The steps for the direct implicit update are described next.

Assume the sampling points $\left(\mathbf{x}_{\mathbf{i}}\right)$ along the crack front and their associated velocities $\left(\mathbf{v}_{\mathbf{i}}\right)$ are given as shown in Fig. 5. The direct update will be applied for the grid nodes that are in a 
specified bandwidth around the crack front. The update bandwidth consists of the quadrilaterals defined by the vertices $\left\{\mathbf{x}_{\mathrm{i}-1}, \mathbf{x}_{\mathrm{i}}, \mathbf{x}_{\mathrm{i}}+\mathbf{v}_{\mathrm{i}}, \mathbf{x}_{\mathrm{i}-1}+\mathbf{v}_{\mathrm{i}-1}\right\}$ for all the sampling points along the crack front. All the grid nodes $\left(\mathbf{p}_{\mathrm{j}}\right)$ within this bandwidth will have a direct update applied.

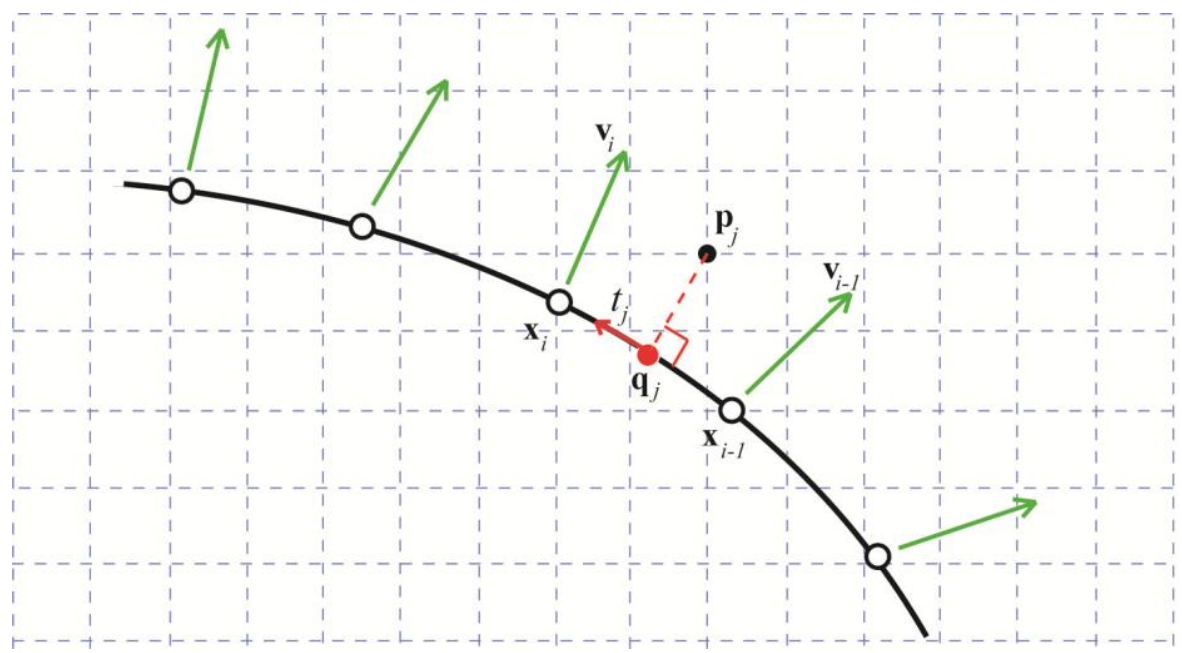

Fig. 5. The sampling points $\left(\mathbf{x}_{\mathrm{i}}\right)$ along the crack front and their associated velocities $\left(\mathbf{v}_{\mathrm{i}}\right)$.

For each of the selected grid nodes, $\mathbf{p}_{\mathrm{j}}$, the nearest point, $\mathbf{q}_{\mathrm{j}}$, on the crack front according to the explicit representation is determined (see Fig. 3).

$$
\mathbf{q}_{j}=(1-\alpha) \mathbf{x}_{i-1}+\alpha \mathbf{x}_{i}
$$

where

$$
\alpha=\frac{\left(\mathbf{p}_{j}-\mathbf{x}_{i-1}\right) \cdot\left(\mathbf{x}_{i}-\mathbf{x}_{i-1}\right)}{\left(\mathbf{x}_{i}-\mathbf{x}_{i-1}\right) \cdot\left(\mathbf{x}_{i}-\mathbf{x}_{i-1}\right)}
$$

The velocity associated with this point is determined by interpolation between the two straddling velocities or by the end velocity if the point is not straddled by two velocities (for example, between the last velocity sample point and the boundary of the solid domain).

$$
\mathbf{v}_{j}=(1-\alpha) \mathbf{v}_{i-1}+\alpha \mathbf{v}_{i}
$$

The tangent vector, $\mathbf{t}_{\mathrm{j}}$, to the crack front at point $\mathbf{q}_{\mathrm{j}}$ can also be obtained from the explicit representation as shown in Fig. 5. Finally, the level set values are updated at each of the selected grid nodes, $\mathbf{p}_{\mathrm{j}}$, as

$$
\begin{aligned}
\phi\left(\mathbf{p}_{j}\right) & =\frac{\mathbf{v}_{j} \times \mathbf{t}_{j}}{\left\|\mathbf{v}_{j} \times \mathbf{t}_{j}\right\|} \cdot\left(\mathbf{p}_{j}-\mathbf{q}_{j}\right) \\
\psi\left(\mathbf{p}_{j}\right) & =\frac{\mathbf{v}_{j}}{\left\|\mathbf{v}_{j}\right\|} \cdot\left(\mathbf{p}_{j}-\mathbf{q}_{j}-\mathbf{v}_{j}\right)
\end{aligned}
$$




\subsection{The explicit approach for tracking cracks}

In the explicit approach, the crack surface is discretized by a triangular mesh (Fig. 6). Rather than incrementally extending the explicit representation, the updated explicit representation is reconstructed from the updated implicit representation each increment. For each cubic element in the implicit grid, triangles for the crack surface are generated using the marching cubes algorithm [19] where the values of the normal level set function $\varphi$ at the grid nodes determine the configuration of triangles. The nodal values of the tangential level set function $\psi$ are then used to determine if any of these triangles intersect the crack front, and thus must be trimmed by cutting them with the triangles generated by the marching cubes algorithm applied to $\psi$. The triangles trimmed in this manner are identified as the new crack front. The marching cubes algorithm was originally developed to create triangle models of constant density surfaces from 3D medical data. It uses a divide-and-conquer approach to generate inter-slice connectivity and creates a case table that defines triangle topology.

To limit the crack surface to the physical problem domain, the surface triangles are also trimmed in the same manner as the crack front by representing the solid domain implicitly by a level set function. The surface triangles trimmed by this process are not considered part of the new crack front as shown in Fig. 6.

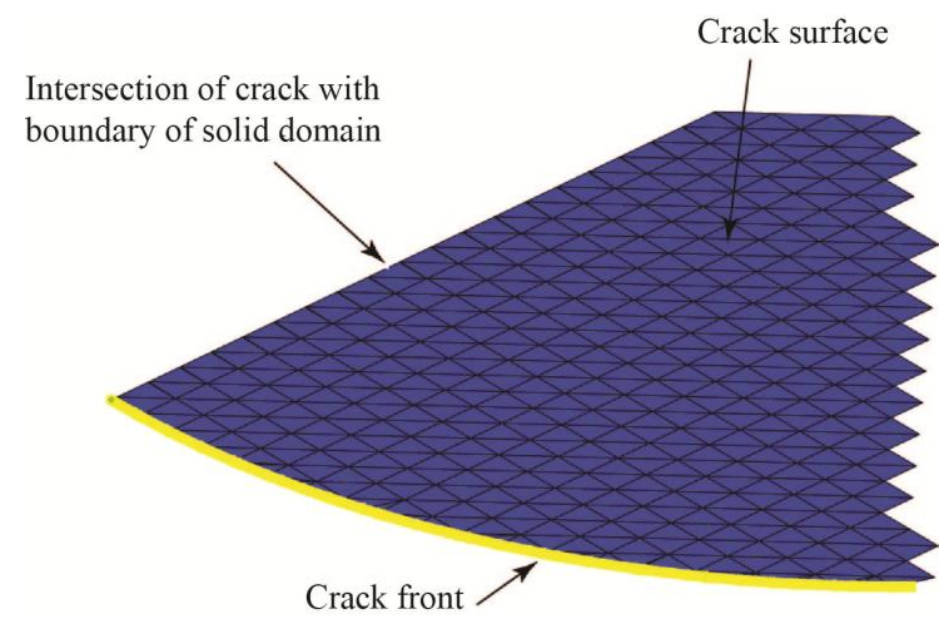

Fig. 6. Explicit representation of crack surface.

\subsection{The hybrid implicit/explicit approach for tracking cracks}

The proposed new hybrid implicit/explicit algorithm is summarized in the flowchart presented in Fig. 7. We assume that the XFEM calculation has produced the sampling points $\mathbf{x}_{\mathbf{i}}$ and their associated velocity data $\mathbf{v}_{\mathbf{i}}$ for the current configuration. The sampling points are first sorted in sequential order along the crack front using the explicit representation so that interpolation between sampling points is efficient, as discussed in Section 3.1. From the explicit crack representation, the crack front location is generated as a parameterized curve. The velocity sampling points $\mathbf{x}_{\mathrm{i}}$ in the implicit representation are then mapped onto the parameterized curve and ordered according to the value of the parameter. 


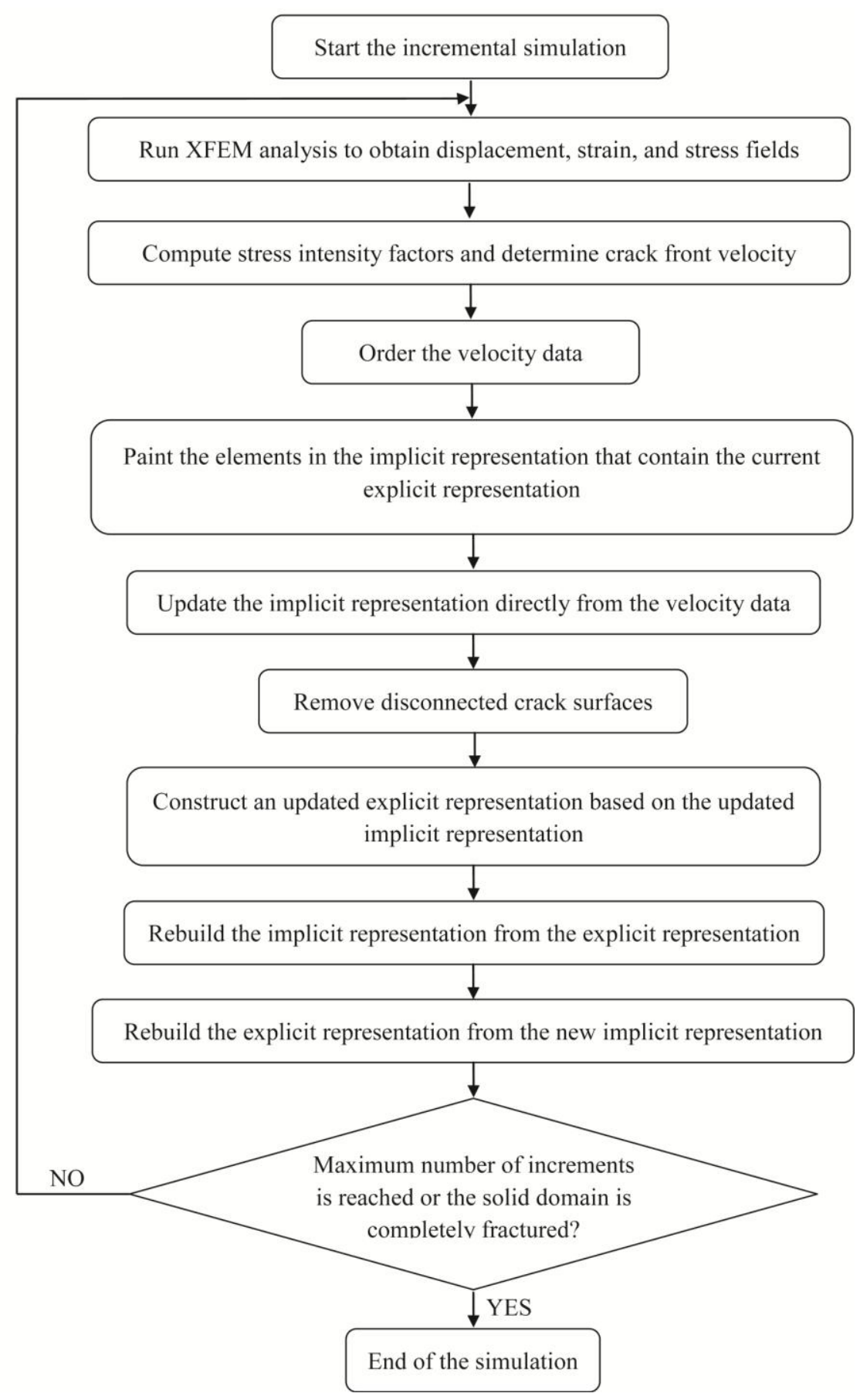

Fig. 7. The proposed algorithm for the hybrid implicit/explicit approach for tracking cracks.

As mentioned earlier, spontaneous creation of spurious crack surfaces far from the true crack surface is a possibility due to the nature of extrapolation. To remove the possibility of these spurious crack surfaces after updating the implicit representation, we need to determine and label the elements in the implicit representation that contain the current explicit representation. These painted elements will be used as a reference to determine spurious crack surfaces after updating the implicit representation. The implicit representation is then directly updated from the velocity 
data based on the algorithm discussed in Section 3.1. To ensure that the new extended crack surface is connected to the previous crack surface, a paint-fill algorithm is employed starting from the points labeled before updating the implicit representation. For these already marked elements, any neighboring element that is also cut by a crack surface is also marked. If a crack surface is disconnected from the previous crack location, then the paint-fill algorithm will not be able to reach that surface and therefore those elements will not be marked. All new crack surfaces that are disconnected are discarded.

In the next step, an updated explicit representation is constructed based on the updated implicit representation as discussed in Section 3.2. A consistent implicit representation is finally rebuilt from the explicit representation. This step is essential to remedy possible discontinuities in the values of the normal level set function $\varphi$ after the direct update of the implicit representation near the crack front. The possibility of such discontinuities arises from the fact that the values of $\varphi$ are not updated behind the crack front, i.e. where $\psi<0$, in the direct update of the implicit representation. Re-building the implicit representation from the explicit representation provides a good reconstruction of the updated crack surface. A continuous formulation for the $\varphi$ and $\psi$ can be computed from the explicit representation by locating the nearest point on the explicit crack surface and using the orientation of the triangles in the explicit representation to determine consistent signs for the value of $\varphi$.

At the end of the current simulation increment, the explicit representation can be re-built from the new implicit representation using the algorithm already discussed for plotting purposes to show where the crack location is at the end of the update step. Obviously, this step is not necessary for the analysis.

The proposed hybrid approach has been implemented into the XFA3D software [11]. XFA3D is an add-on toolkit for Abaqus which performs XFEM simulations through user-defined elements in Abaqus. Using XFA3D we are able to insert cracks into the base Abaqus model. Based on the size and location of the embedded crack, an enriched zone through user-defined elements is defined around the crack, allowing the discontinuity to be modeled using the XFEM technique. In the numerical simulations, crack growth is controlled based on the stress intensity factors $\left(K_{\mathrm{I}}\right.$, $K_{\mathrm{II}}$, and $\left.K_{\mathrm{III}}\right)$ along the crack front. The stress intensity factors are calculated using crack front opening displacements as

$$
\begin{aligned}
& K_{I}=\mu \sqrt{\frac{2 \pi}{r}} \frac{\delta_{1}}{\kappa+1} \\
& K_{I I}=\mu \sqrt{\frac{2 \pi}{r}} \frac{\delta_{2}}{\kappa+1} \\
& K_{I I I}=\mu \sqrt{\frac{2 \pi}{r}} \frac{\delta_{3}}{4}
\end{aligned}
$$

in which $\mu$ is the shear modulus, $r$ is the distance between the point at which the opening displacement is computed and the crack front, $\delta_{1}, \delta_{2}$, and $\delta_{3}$ are the crack front opening displacements in the familiar local coordinates at the crack front, and $\kappa=3-4 v$ where $v$ is the Poisson's ratio. The local coordinate system $\left(\mathbf{e}_{1}, \mathbf{e}_{2}, \mathbf{e}_{3}\right)$ at the crack front is shown in Fig. 8 where $\mathbf{e}_{1}$ is the unit normal to the current crack surface pointing to positive normal level set 
values $\varphi, \mathbf{e}_{2}$ is normal to the current crack front, tangent to the crack surface, and pointing away from the interior of the crack, and $\mathbf{e}_{3}=\mathbf{e}_{1} \quad \mathbf{e}_{2}$ is tangent to the crack front.

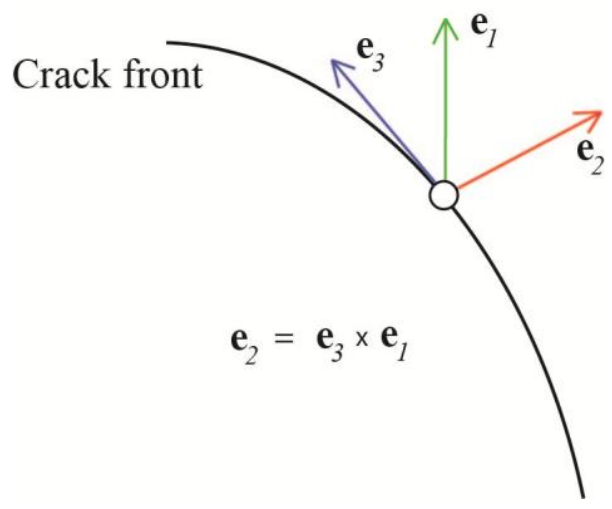

Fig. 8. The local coordinate system $\left(\mathbf{e}_{1}, \mathbf{e}_{2}, \mathbf{e}_{3}\right)$ at the crack front. The vector $\mathbf{e}_{1}$ is the unit normal to the current crack surface pointing to positive normal level set values $\varphi, \mathbf{e}_{2}$ is normal to the current crack front, tangent to the crack surface, and pointing away from the interior of the crack, and $\mathbf{e}_{3}=\mathbf{e}_{1} \quad \mathbf{e}_{2}$ is tangent to the crack front.

For mixed mode crack growth, the equivalent stress intensity factor is calculated as

$$
K_{\text {eqv }}=\sqrt{K_{I}^{2}+K_{I I}^{2}+K_{I I I}^{2}(1+v)}
$$

The crack growth is assumed to be on the $\left\{\mathbf{e}_{1}, \mathbf{e}_{2}\right\}$ plane and the angle between the growth direction and $\mathbf{e}_{2}$ is determined based on the maximum hoop stress criterion as

$$
\begin{aligned}
& \theta=2 \operatorname{atan}\left(\frac{K_{I}}{4 K_{I I}}-\frac{1}{4} \sqrt{\left(\frac{K_{I}}{K_{I I}}\right)^{2}+8}\right), \text { for } K_{I I}>0 \\
& \theta=2 \operatorname{atan}\left(\frac{K_{I}}{4 K_{I I}}+\frac{1}{4} \sqrt{\left(\frac{K_{I}}{K_{I I}}\right)^{2}+8}\right), \text { for } K_{I I}<0
\end{aligned}
$$

Finally, the crack growth size is computed from the Paris step

$$
\frac{d a}{d N}=C(\Delta K)^{m}
$$

where $a$ is the crack length, $N$ is the number of cycles, $C$ and $m$ are the fatigue parameters, and $\Delta K$ is the range of equivalent stress intensity factors.

\section{Numerical examples}


In this section, effectiveness of the proposed approach is demonstrated through four numerical examples: (1) a quarter-circular crack in a complex helicopter component, for which employing the implicit crack representation leads to the creation of spurious crack surfaces while the crack propagation can be modeled successfully with the proposed hybrid approach, (2) a U-shape crack in a cuboid which demonstrates the difficulty associated with the explicit crack representation in modeling merging crack velocity vectors, (3) an inclined elliptical crack in a cuboid, and (3) a beam under three-point bending load with an inclined notch plane. The last two examples demonstrate the capability of the proposed hybrid approach in modeling non-planar 3D crack growth.

\subsection{Quarter-circular crack in a complex helicopter component}

This round-robin challenge problem has already been studied by Newman Jr. et al. [21] and Shi et al. [11]. Fig. 9 shows the Abaqus discretized model of this problem, consisting of a flanged plate with a central lightening hole. The UEL zone, called the XFEM zone, is also shown in this figure. The user-defined elements are introduced in the XFEM zone while the rest of the problem domain is modeled using conventional Abaqus solid elements. The plate is aluminum alloy of AA 7010 T73651. More details about this example can be found in [11, 21]. This example is representative of many features found in a helicopter lift frame. Initial crack is a corner defect (2 $\mathrm{mm}$ in radius) at the edge of the central hole as shown in Fig. 9.

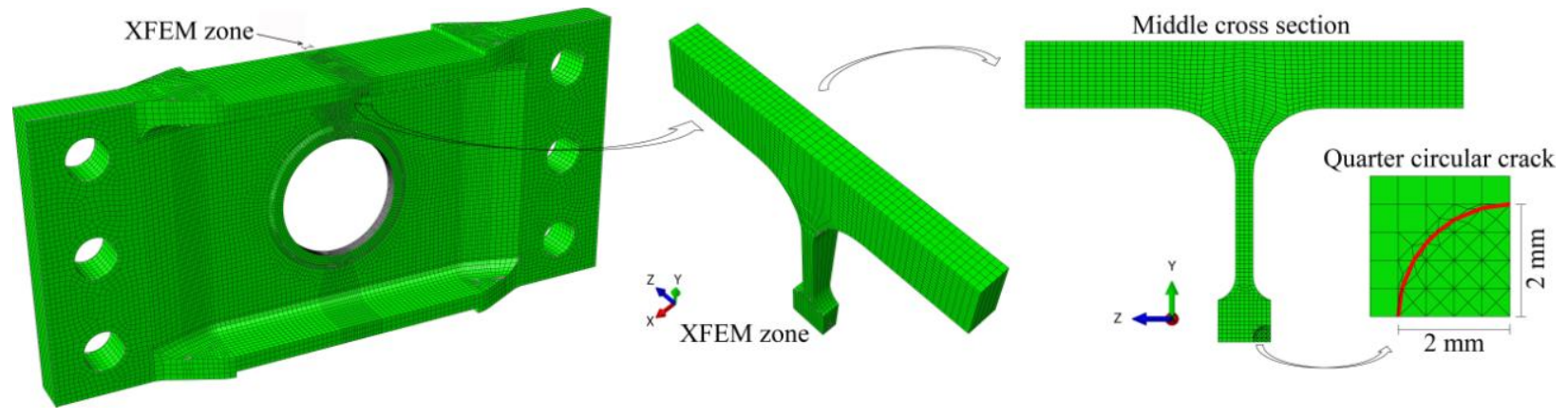

Fig. 9. The discretized model for the helicopter component.

To simulate the crack propagation, we first used XFA3D with the implicit crack representation. As shown in Fig 3, this simulation lead to the creation of a spurious crack surface, which is disconnected from the physical crack surface. Then, we repeat the simulation using the hybrid implicit/explicit crack representation in XFA3D, resulting in successful modeling of the crack propagation in the plate. Eight snapshots of the crack propagation are shown in Fig. 10. 

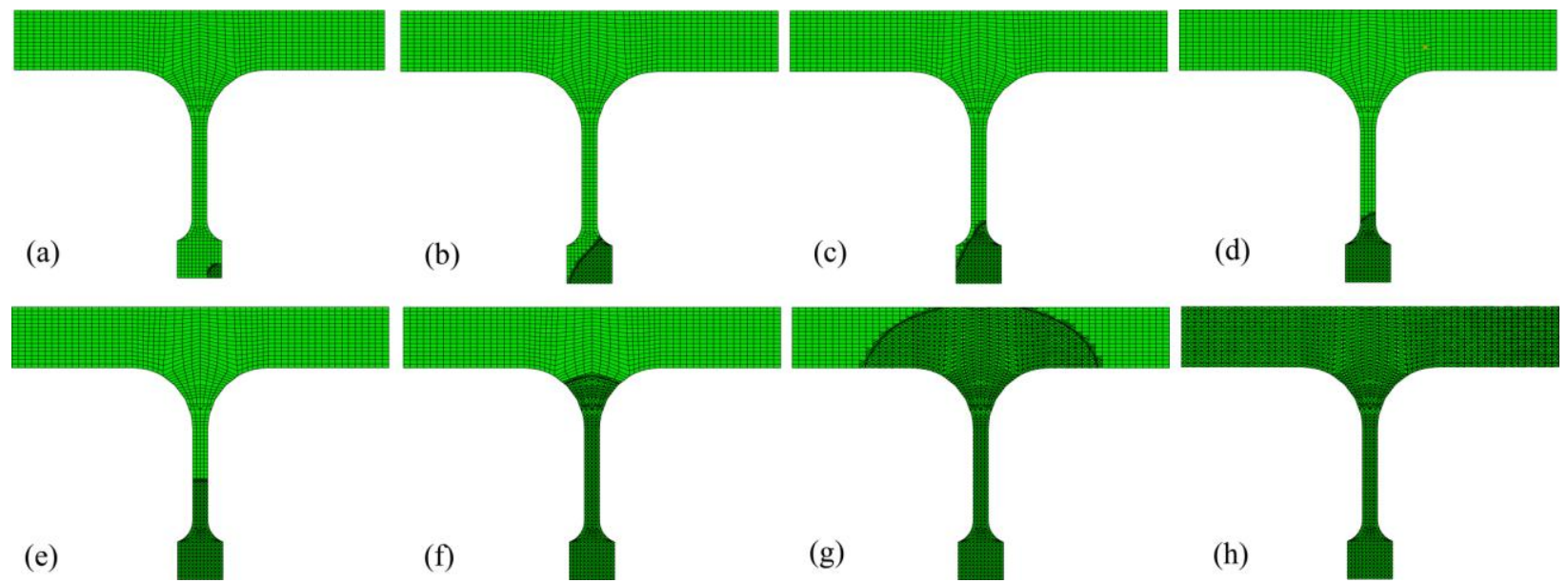

Fig. 10. Eight snapshots of the crack propagation in the helicopter component.

\subsection{U-shaped crack in a cuboid}

To demonstrate the advantage of the proposed approach over the explicit crack representation, crack propagation from a U-shape crack in a cuboid is modeled using the XFA3D with (1) the explicit and (2) the proposed hybrid implicit/explicit representations of the crack. In this example, an initial U-shape crack is embedded in a cuboid of size $2.0 \times 2.0 \times 1.0 \mathrm{~m}^{3}$, which is subjected to a uniform tensile traction of $100 \mathrm{~Pa}$ as shown in Fig. 11a. The discretized finite element model and the XFEM zone are shown in Fig. $11 \mathrm{~b}$ and 11c. The modulus of elasticity and Poisson's ratio are $E=3 \times 10^{10} \mathrm{~Pa}$ and $v=0.3$ respectively. The fatigue parameters are taken as $C=3.16 \times 10^{-14}$ and $m=3.0$ where the growth rate and the stress intensity factor are in $\mathrm{m} /$ cycle and $P a \sqrt{m}$ respectively; and the load ratio is assumed to be $R=0.1$.

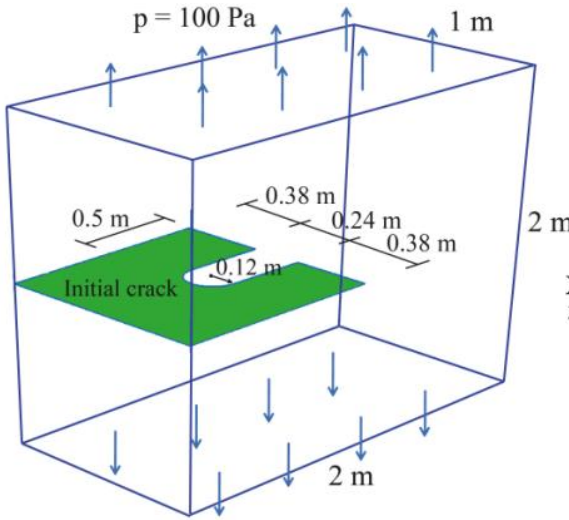

(a)

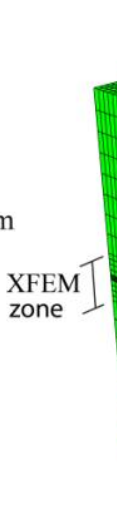

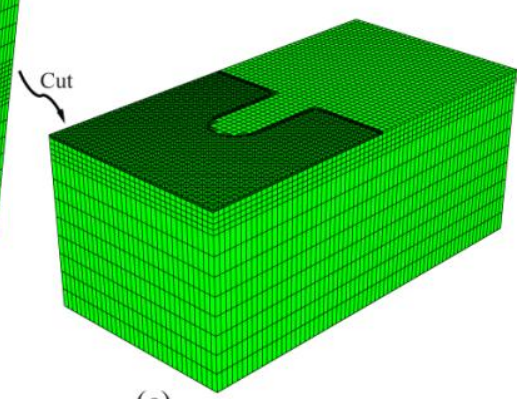

(c)

Fig. 11. (a) Geometry and (b-c) the discretized model for the U-shape crack example.

As mentioned in Section 1, XFA3D with the explicit crack representation failed to model the crack propagation (see Fig. 4). Here, we repeat the simulation using the hybrid implicit/explicit crack representation in XFA3D. Fig. 12, showing eight snapshots of the crack propagation, shows that XFA3D with the hybrid implicit/explicit crack representation results in successful modeling of the crack propagation. 


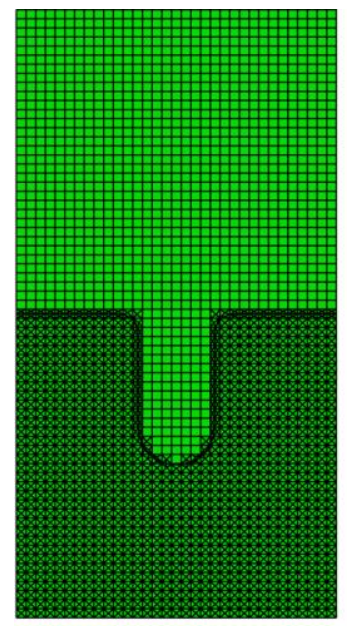

(a)

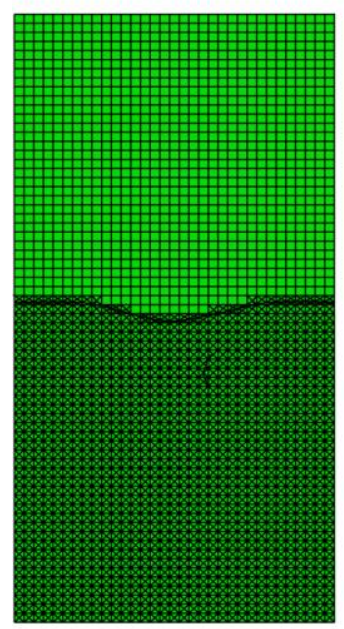

(e)

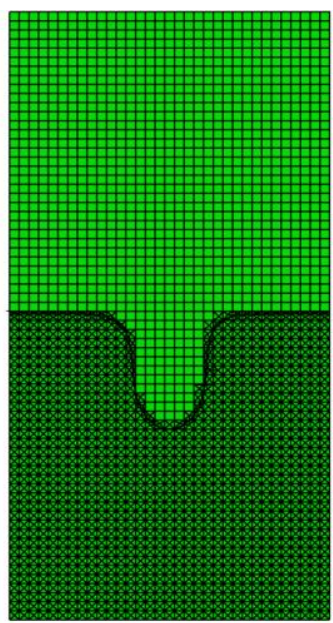

(b)

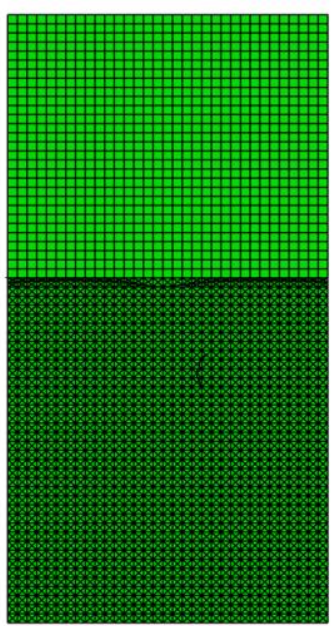

(f)

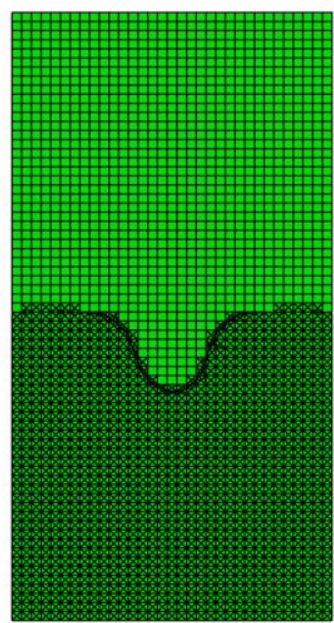

(c)

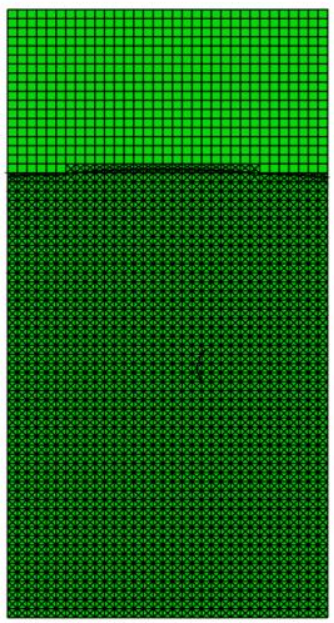

(g)

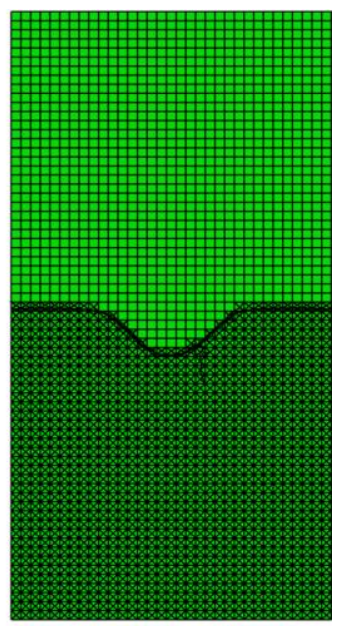

(d)

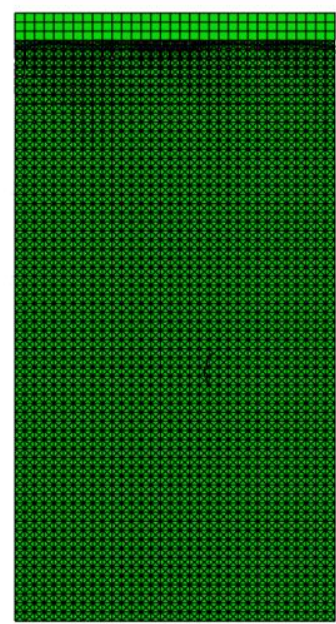

(h)

Fig. 12. Eight snapshots of the crack propagation in the U-shape crack example.

\subsection{Inclined elliptical crack in a cuboid}

To evaluate the effectiveness of the proposed hybrid approach for tracking 3D non-planar cracks, crack propagation from an initial inclined elliptical flaw is simulated. The problem consists of an inclined elliptical crack of dimensions $2 a=0.384 \mathrm{~m}$ and $2 b=0.192 \mathrm{~m}$ embedded in a cuboid of edge sizes $0.6 \times 0.6 \times 0.4 \mathrm{~m}^{3}$, as illustrated in Fig. 13. The slope of the crack with respect to the $\mathrm{y}$-axis is 30 degrees. The domain is subjected to a uniform tensile traction of $4.275 \mathrm{MPa}$ in the $\mathrm{z}-$ axis. The discretized finite element model and the XFEM zone are shown in Fig. 14. The material parameters used in this analysis are: modulus of elasticity, $E=7 \times 10^{4} \mathrm{MPa}$, and Poisson's ratio, $v=0.33$. The fatigue parameters are taken as $C=6.442 \times 10^{-28}$ and $m=3.4073$ where the growth rate and the stress intensity factor are in $\mathrm{m} /$ cycle and $\mathrm{Pa} \sqrt{\mathrm{m}}$ respectively; and the load ratio is assumed to be $R=-1.0$. 


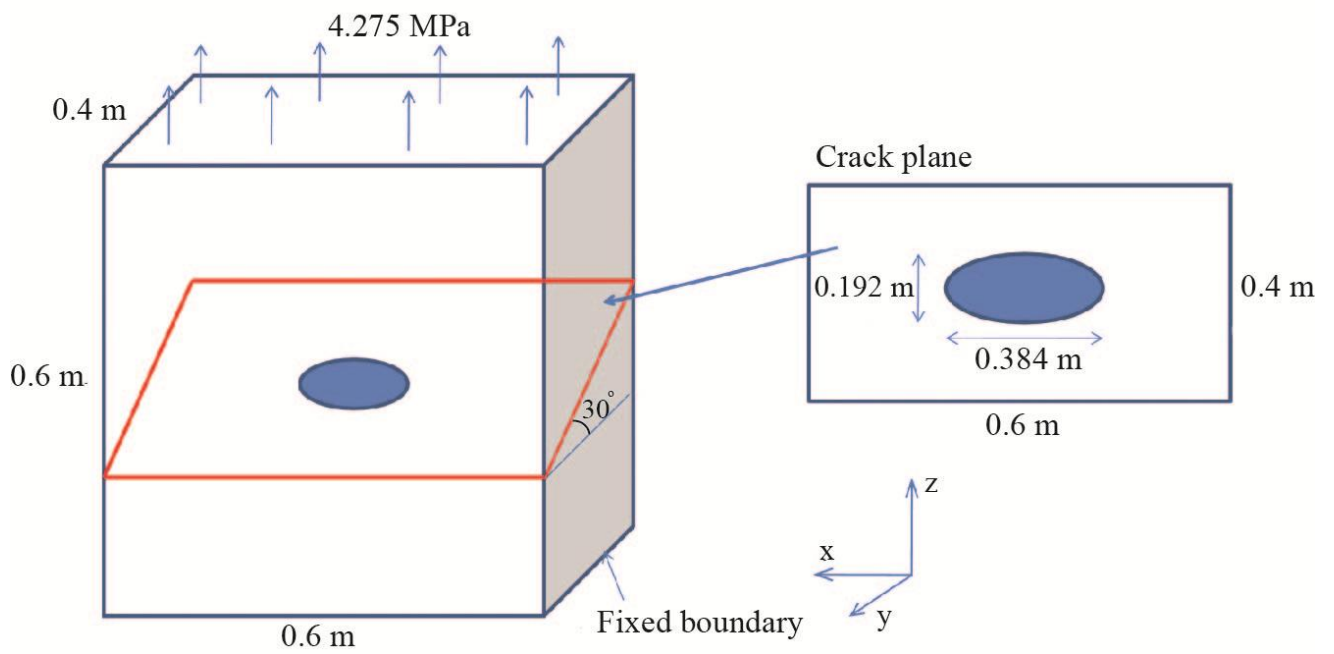

Fig. 13. Geometry of the inclined elliptical crack example.

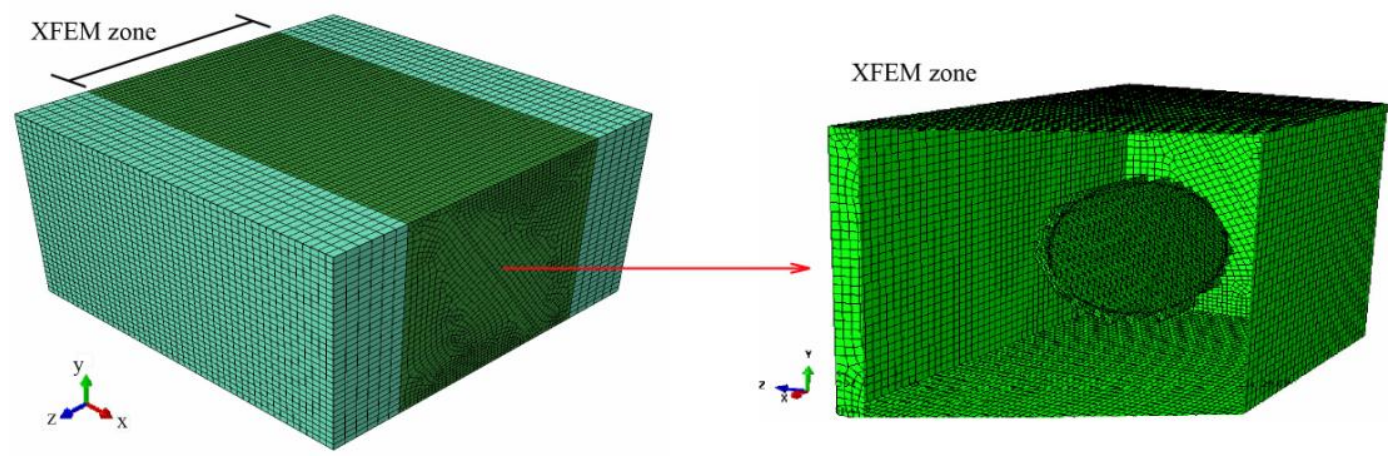

Fig. 14. Discretized model for the inclined elliptical crack example.

The analytical solutions of the stress intensity factors for modes I, II, and III of an inclined elliptical crack embedded in an infinite domain [22] are used as reference to evaluate the accuracy of the numerical K-prediction at the initial configuration. A comparison between the numerical K prediction at the initial configuration with the analytical solution is presented in Fig. 15. There is a small discrepancy between the numerical and analytical $K_{I}$ values which is due to the finite domain effects in the numerical model while the analytical solution is associated with an infinite domain. To show the accuracy of K-prediction in our numerical model, we repeated the numerical model with the same crack but an expanded domain size of $4.0 \times 4.0 \times 4.0 \mathrm{~m}^{3}$. The obtained $\mathrm{K}$ values for the expanded domain are also shown in Fig. 15, which are in a very good agreement with the analytical solutions. Finally, we employed XFA3D for crack propagation in this example. Fig. 16 shows the final crack surface which is propagated as expected. 


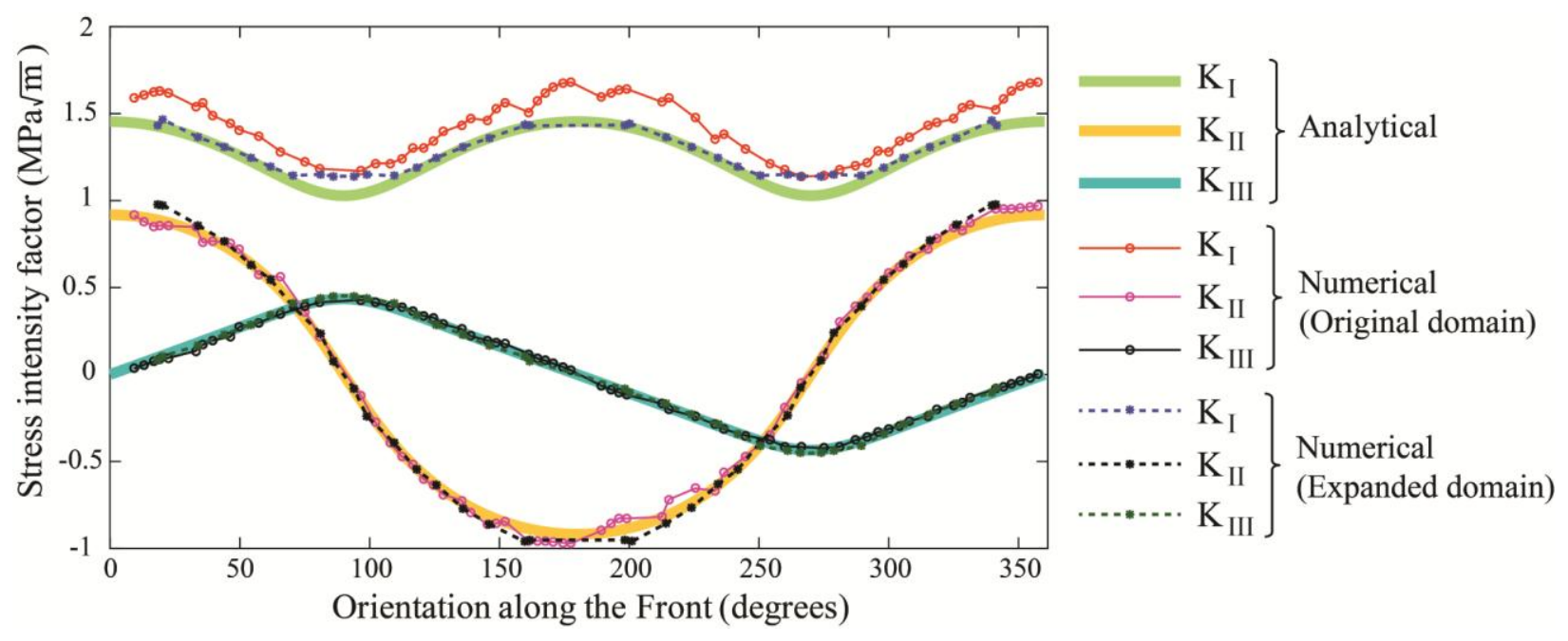

Fig. 15. Comparison between numerical K prediction at the initial configuration with the analytical solution for the inclined elliptical crack example.

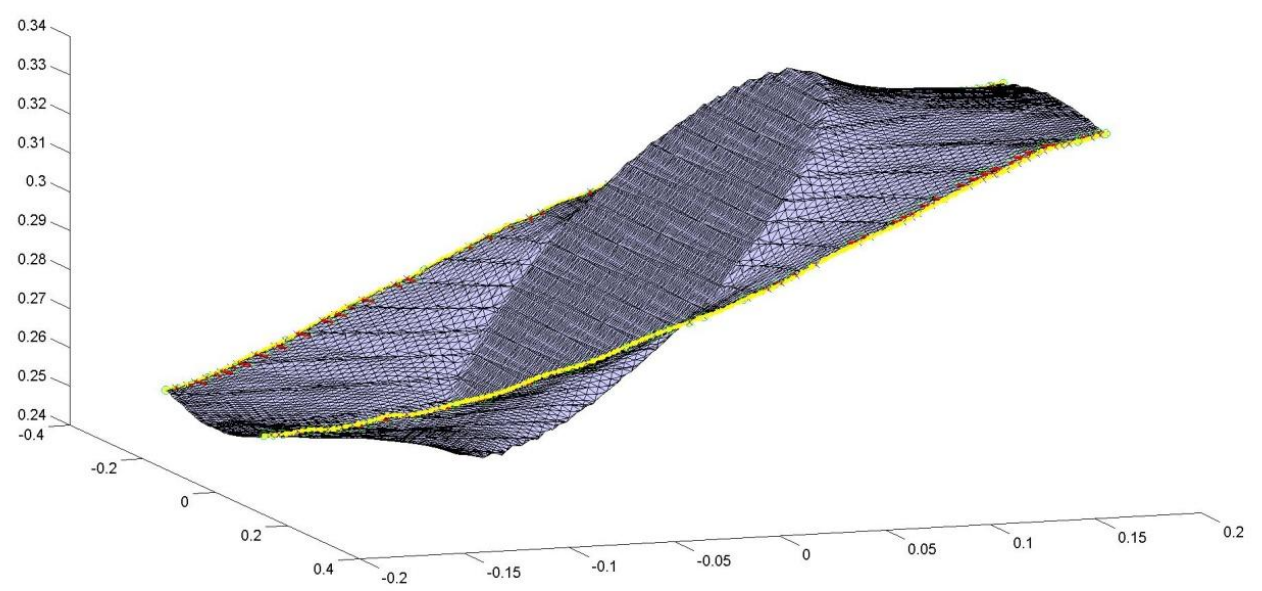

Fig. 16. Final crack surface for the inclined elliptical crack example.

\subsection{Inclined notch in a three-point bending beam}

In this example, the single edge notched specimen with a crack inclined with respect to the midplane between its two supports is considered (Fig. 17). The specimen is subject to three point bending. The experimental results for this example are presented in [23]. The specimen size is $260 \times 60 \times 10 \mathrm{~mm}^{3}$, the applied force is $F=2 \mathrm{kN}$ per $\mathrm{mm}$ thickness as shown in Fig. 17. The initial crack length is $20 \mathrm{~mm}$ and is inclined with respect to the mid-plane with the angle of 45 degrees. The discretized finite element model and the XFEM zone are shown in Fig. 18. The modulus of elasticity is $E=2.1 \times 10^{5} \mathrm{MPa}$ and Poisson's ratio is $v=0.3$. The fatigue parameters are taken as $C=4.515 \times 10^{-14}$ and $m=3.531$ where the growth rate and the stress intensity factor are in $\mathrm{m} /$ cycle and $\mathrm{Pa} \sqrt{\mathrm{m}}$ respectively; and the load ratio is assumed to be $R=0.1$. 


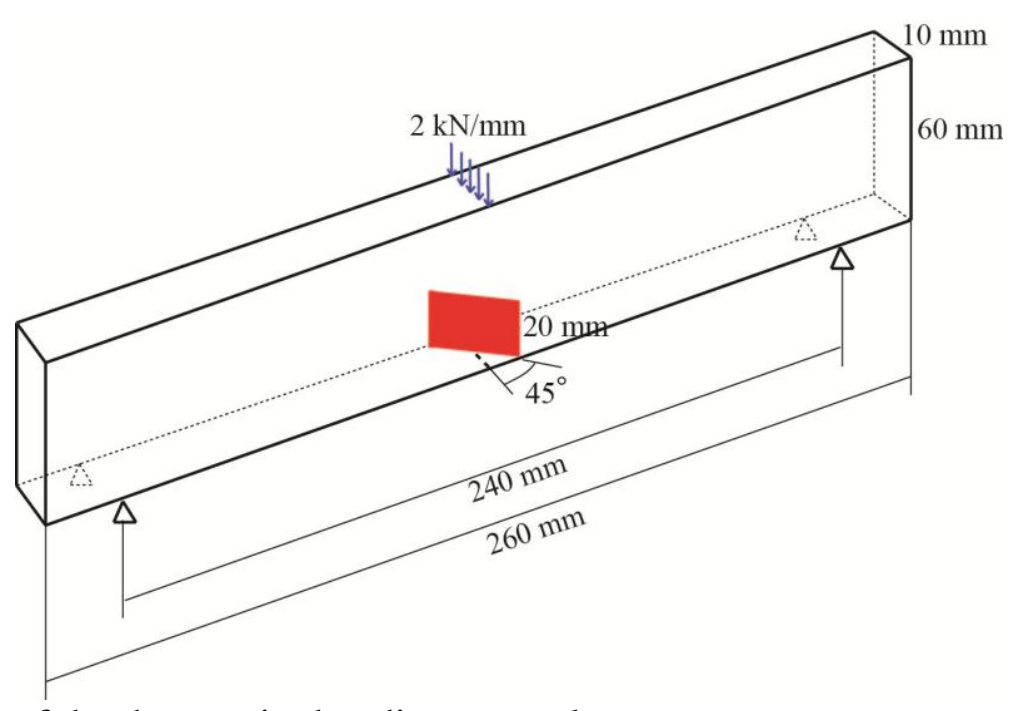

Fig. 17. Geometry of the three-point bending example.

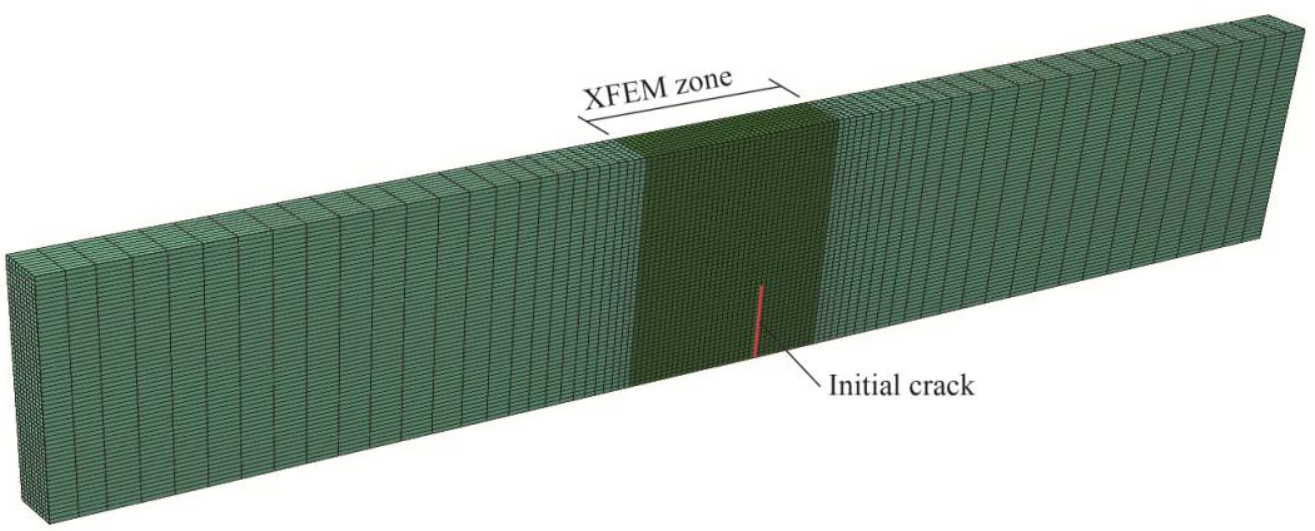

Fig. 18. Discretized finite element model and the XFEM zone in the three-point bending example.

The final configuration of the crack in the XFEM zone and two views of the final crack surface are depicted in Fig. 19. The final crack surface presented in this figure is qualitatively in good agreement with the experimental results presented in [23]. 

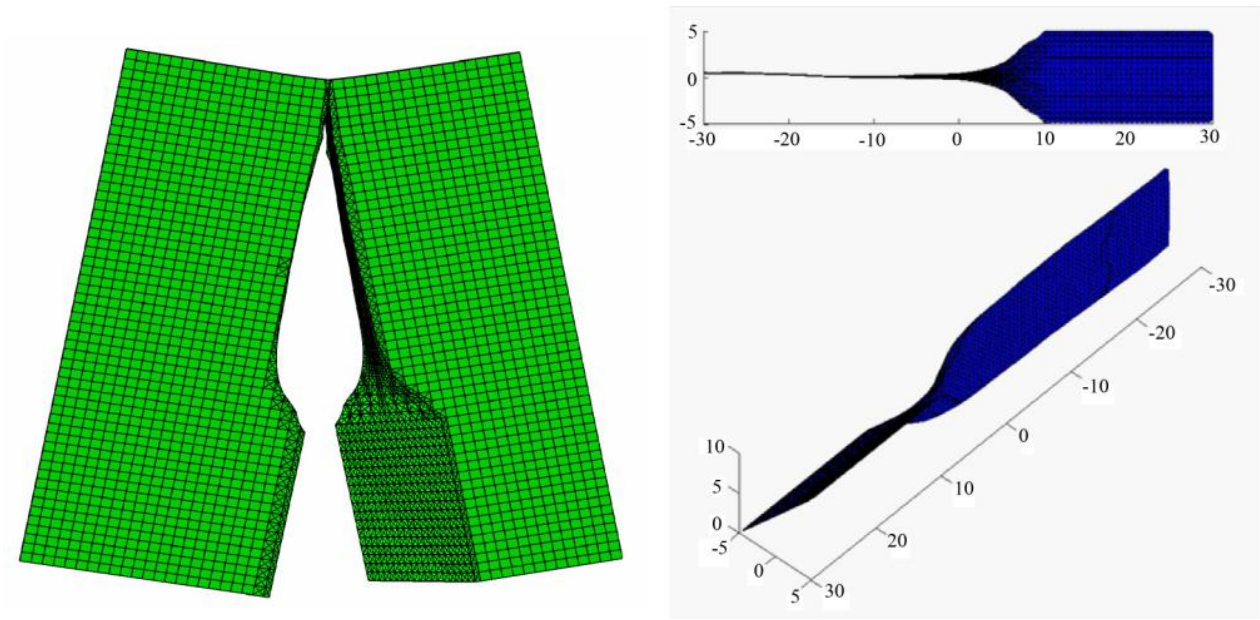

Fig. 19. Final configuration of the crack in the three-point bending example, which is in good agreement with the experimental results presented in [23].

\section{Conclusions}

In the XFEM, the crack surface can be described by either the implicit representation or the explicit representation. To take advantage of the strengths of each, while simultaneously covering their weaknesses, we developed and implemented a hybrid implicit/explicit approach for tracking cracks in the XFEM simulations in our extended finite element toolkit for Abaqus (XFA3D). The implicit representation through the use of the level set method in the XFEM is becoming a standard because of the important advantages that the level set method provides for the XFEM. The level set functions imply the local coordinate system required for the evaluation of the enrichment functions and it is also used in stress intensity factor computations and for the determination of fully/partially cut elements by the crack. Furthermore, the level set method is convenient for handling situations where the crack front is concave and the velocity vectors may cross. However, the implicit representation encounters some challenges such as when spurious cracks may arise that are erroneously created in regions disconnected and often far away from the true crack surface. On the other hand, the crack geometry can be described by an explicit triangulated mesh which can be easily updated after a propagation step, and it can clearly demarcate the location of the crack surface and front. But, the explicit representation has difficulties in for example handling crack overlaps and extraction of crack local coordinates. We notice that although the proposed hybrid approach does not have the drawbacks of the implicit and explicit representations, it is clearly more computationally demanding which may impact the computational efficiency for a large scale problem.

In the proposed hybrid approach, the implicit representation is updated directly from the crack front velocity data after each propagation step. The normal level set values are updated only ahead of the crack front. For the updated implicit representation, there is a possibility that new crack surfaces disconnected to the current surface can appear due to extrapolation away from the crack front. The explicit representation ensures that all new disconnected crack surfaces are removed. Then, an updated explicit representation is constructed based on the updated implicit representation using the marching cubes algorithm. Finally, the implicit representation is rebuilt 
from the explicit representation to ensure that the data in the level set representation is generated from a consistent crack description. The proposed hybrid approach has been implemented in a 3D extended finite element toolkit for Abaqus (XFA3D). The effectiveness of the proposed approach for non-planar crack propagation is demonstrated through four numerical examples: a quarter-circular crack in a complex helicopter component, a u-shaped crack and an inclined elliptical crack in cuboids, and an inclined edge crack in a three-point bending beam. Future study will be performed by enhancing the efficiency of XFA3D by parallelization of userdefined XFEM elements in Abaqus.

\section{Acknowledgement}

The authors are grateful for the support provided by the Office of Naval Research (N00014-13C-0108) for which Dr. Paul Hess serves as the technical monitor. We also would like to thank Mr. Yared Amanuel at Code 65 of NSWCCD for technical discussion and evaluation of our XFA3D toolkit.

\section{References}

[1] Belytschko, T., \& Black, T. (1999). Elastic crack growth in finite elements with minimal remeshing. International journal for numerical methods in engineering, 45(5), 601-620.

[2] Dolbow, J., \& Belytschko, T. (1999). A finite element method for crack growth without remeshing. International journal for numerical methods in engineering, 46(1), 131-150.

[3] Fries, T. P., \& Belytschko, T. (2010). The extended/generalized finite element method: an overview of the method and its applications. International Journal for Numerical Methods in Engineering, 84(3), 253-304.

[4] Sukumar, N., Moës, N., Moran, B., \& Belytschko, T. (2000). Extended finite element method for three-dimensional crack modelling. International Journal for Numerical Methods in Engineering, 48(11), 1549-1570.

[5] Moës, N., \& Belytschko, T. (2002). Extended finite element method for cohesive crack growth. Engineering fracture mechanics, 69(7), 813-833.

[6] Moës, N., Gravouil, A., \& Belytschko, T. (2002). Non-planar 3D crack growth by the extended finite element and level sets-Part I: Mechanical model. International Journal for Numerical Methods in Engineering, 53(11), 2549-2568.

[7] Gravouil, A., Moës, N., \& Belytschko, T. (2002). Non-planar 3D crack growth by the extended finite element and level sets-Part II: Level set update. International Journal for Numerical Methods in Engineering, 53(11), 2569-2586.

[8] Sukumar, N., \& Prévost, J. H. (2003). Modeling quasi-static crack growth with the extended finite element method Part I: Computer implementation. International journal of solids and structures, 40(26), 7513-7537. 
[9] Huang, R., Sukumar, N., \& Prévost, J. H. (2003). Modeling quasi-static crack growth with the extended finite element method Part II: Numerical applications. International Journal of Solids and Structures, 40(26), 7539-7552.

[10] Areias, P., \& Belytschko, T. (2005). Analysis of three-dimensional crack initiation and propagation using the extended finite element method. International Journal for Numerical Methods in Engineering, 63(5), 760-788.

[11] Shi, J., Chopp, D., Lua, J., Sukumar, N., \& Belytschko, T. (2010). Abaqus implementation of extended finite element method using a level set representation for three-dimensional fatigue crack growth and life predictions. Engineering Fracture Mechanics, 77(14), 2840-2863.

[12] Silling, S. A. (2000). Reformulation of elasticity theory for discontinuities and long-range forces. Journal of the Mechanics and Physics of Solids, 48(1), 175-209.

[13] Silling, S. A., Epton, M., Weckner, O., Xu, J., \& Askari, E. (2007). Peridynamic states and constitutive modeling. Journal of Elasticity, 88(2), 151-184.

[14] Chen, H., Lin, E., \& Liu, Y. (2014). A novel Volume-Compensated Particle method for 2D elasticity and plasticity analysis. International Journal of Solids and Structures, 51(9), 1819-1833.

[15] Chen, H., Lin, E., Jiao, Y., \& Liu, Y. (2014). A generalized 2D non-local lattice spring model for fracture simulation. Computational Mechanics, 54(6), 1541-1558.

[16] Stolarska, M., Chopp, D. L., Moës, N., \& Belytschko, T. (2001). Modelling crack growth by level sets in the extended finite element method. International journal for numerical methods in Engineering, 51(8), 943-960.

[17] Belytschko, T., Parimi, C., Moës, N., Sukumar, N., \& Usui, S. (2003). Structured extended finite element methods for solids defined by implicit surfaces. International journal for numerical methods in engineering, 56(4), 609-635.

[18] Sukumar, N., Chopp, D. L., \& Moran, B. (2003). Extended finite element method and fast marching method for three-dimensional fatigue crack propagation. Engineering Fracture Mechanics, 70(1), 29-48.

[19] Lorensen, W. E., \& Cline, H. E. (1987). Marching cubes: A high resolution 3D surface construction algorithm. ACM siggraph computer graphics 21(4), 163-169.

[20] Fries, T. P., \& Baydoun, M. (2012). Crack propagation with the extended finite element method and a hybrid explicit-implicit crack description. International Journal for numerical methods in engineering, 89(12), 1527-1558.

[21] Newman, J. C., Irving, P. E., Lin, J., \& Le, D. D. (2006). Crack growth predictions in a complex helicopter component under spectrum loading. Fatigue \& fracture of engineering materials \& structures, 29(11), 949-958.

[22] Tada H., Paris P., \& Irwin G. The Stress Analysis of Cracks Handbook. ASME Press, New York, 3rd edition, 2000. 
[23] Buchholz, F. G., Chergui, A., \& Richard, H. A. (2004). Fracture analyses and experimental results of crack growth under general mixed mode loading conditions. Engineering Fracture Mechanics, 71(4), 455-468. 